\title{
Evaluación de la revisión de transcripciones y traducciones automáticas de vídeos poliMedia*
}

\author{
Juan Daniel Valor Miró ${ }^{1}$, Carlos Turró ${ }^{1}$, Jorge Civera ${ }^{1}$ y Alfons Juan ${ }^{1}$ \\ ${ }^{1}$ Universitat Politècnica de València
}

\begin{abstract}
Resumen
Los vídeos docentes son una herramienta de gran aceptación en el mundo universitario, que se integran en metodologías activas y innovadoras, dando pie a plataformas como poliMedia, plataforma de la Universitat Politècnica de València (UPV) que permite la creación, publicación y difusión de este tipo de contenido multimedia. En el marco del proyecto europeo transLectures, la UPV ha generado automaticamente transcripciones y traducciones en español, catalán e inglés para todos los vídeos incluidos en el repositorio poliMedia.

Las transcripciones y traducciones automáticas generadas tienen una alta calidad. Sin embargo, estas transcripciones y traducciones poseen errores inherentes al proceso automático de subtitulado utilizado, por lo que en ocasiones puede ser necesaria una revisión manual posterior para garantizar la ausencia de errores. El objetivo de este trabajo es evaluar este proceso de revisión manual para poder compararlo en términos de coste temporal con un proceso de obtención completamente manual. Así pues, en el marco de las ayudas de la UPV de Docencia en Red 2013-2014, pusimos en marcha una campaña de evaluación del proceso de revisión de transcripciones y traducciones automáticas por parte del profesorado, cuyos resultados indican inequívocamente la eficacia de estas técnicas y el importante ahorro de tiempo que suponen.
\end{abstract}

Keywords: transcripciones, traducciones, evaluaciones con usuario, docencia en red, poliMedia

\footnotetext{
* The research leading to these results has received funding from the European Union FP7/20072013 under grant agreement no 287755 (transLectures) and ICT PSP/2007-2013 under grant agreement no 621030 (EMMA), and the Spanish MINECO Active2Trans (TIN2012-31723) research project.
} 


\section{Introducción}

Los vídeos docentes son una herramienta que cada vez tienen una mayor aceptación en el mundo universitario y que permite su integración en metodologías activas e innovadoras dando lugar a plataformas como poliMedia (Turró y col. 2009), plataforma de la Universitat Politècnica de València (UPV) que permite la creación, publicación y difusión de vídeos docentes. En el marco del proyecto europeo transLectures (Silvestre y col. 2012), con el objetivo de generar transcripciones y traducciones de forma automatizada en español, catalán e inglés para todos los vídeos incluidos en el repositorio poliMedia, mediante el uso de técnicas estadísticas de aprendizaje automático (Valor Miró y col. 2012).

poliMedia es un sistema diseñado por la UPV para la creación de contenidos multimedia de apoyo a la docencia presencial (Turró y col. 2009). Este sistema comprende desde la preparación de los vídeos docentes hasta la distribución de los mismos por distintos canales de comunicación. De esta forma, a través de poliMedia, la UPV ofrece instrumentos, materiales y personal técnico para dar soporte a la grabación de los vídeos docentes por parte del profesorado. Estos vídeos docentes siguen un formato estándar característico de la plataforma. Se trata de una vista conjunta del profesor y de la pantalla de un ordenador con diapositivas, programas, etc, en un mismo plano de cámara fijo en todo el vídeo, como se puede observar en la Figura 1.

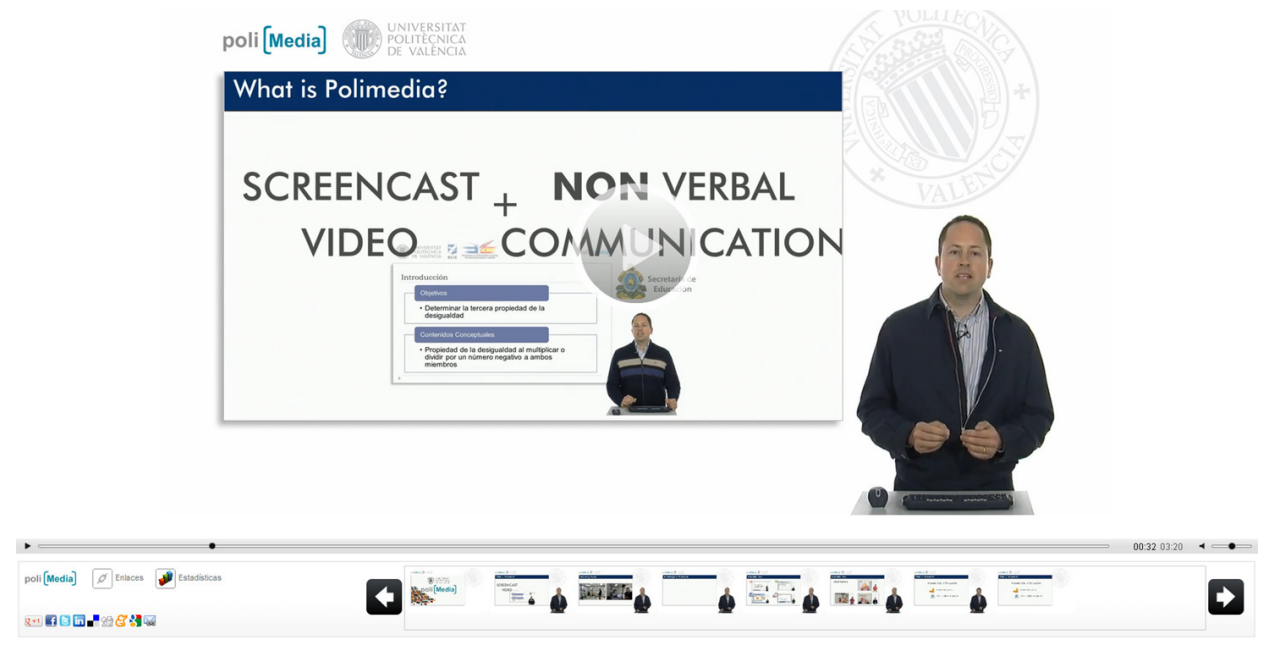

Fig. 1: Resultado final de un vídeo docente de poliMedia.

El proceso de publicación de vídeos docentes es muy simple: se graba simultáneamente en un estudio al profesor sobre un fondo blanco, y la pantalla del ordenador que éste mismo emplea durante el vídeo. Después se extrae la grabación del profesor y se combina con la de la pantalla. Finalmente, este vídeo ya editado se distribuye a través del portal oficial de poliMedia, y de otros canales de distribución. 
Por otro lado, transLectures es el acrónimo del proyecto europeo "Transcription and Translation of Video Lectures" (2011-2014), en el cual se aplican técnicas de reconocimiento automático del habla y de traducción automática con el objetivo de proveer de transcripciones y traducciones automáticas a grandes repositorios de vídeos docentes como poliMedia (Silvestre y col. 2012).

\section{Objetivos}

Las transcripciones y traducciones automáticas generadas tienen una alta calidad, pero en cualquier caso, estas transcripciones automáticas poseen errores inherentes al proceso de subtitulado automático utilizado, por lo que es necesaria una revisión manual posterior para garantizar una calidad adecuada para el alumnado. El objetivo de este trabajo es evaluar este proceso de revisión manual a partir de transcripciones y traducciones automáticas, para poder compararlo en términos de coste temporal con un proceso de obtención totalmente manual. Así pues, en el marco de las ayudas de la UPV de Docencia en Red 2013-2014, y tras el éxito de la edición anterior (Valor Miró y col. 2014a), pusimos en marcha un proceso de revisión de transcripciones y traducciones automáticas por parte del profesorado de diversos vídeos docentes de poliMedia. Para ello, empleando un proceso de posedición (Valor Miró y col. 2014b), hemos realizado una evaluación de la revisión de subtítulos disponibles en los diferentes idiomas.

\section{Desarrollo de la innovación}

Se ha realizado una evaluación del proceso de revisión de transcripciones y traducciones de vídeos docentes, empleando una plataforma pionera de reciente integración en poliMedia, la plataforma transLectures.

En las evaluaciones han participado profesores que previamente habían grabado material para el portal poliMedia y otros profesores voluntarios. Estas revisiones se han llevado a cabo en el marco del programa Docència en Xarxa, que pretende incentivar la adopción de las nuevas tecnologías en la elaboración de materiales docentes por parte del profesorado. Así pues, se ha realizado un seguimiento y evaluación de la revisión de transcripciones en español, catalán, e inglés, y de la supervision de traducciones del español al inglés; por parte del profesorado de la UPV.

Para realizar estas revisiones se ha diseñado un reproductor de vídeo web (Valor Miró y col. 2012) que permite la modificación de los subtítulos mediante una interfaz testeada en la edición anterior de Docència en Xarxa (Valor Miró y col. 2014a). Este reproductor de vídeo puede verse en funcionamiento en la Figura 2. Concretamente se han revisado las transcripciones de 135 vídeos en español, 57 en inglés, y 19 en catalán; así como las traducciones del español al inglés de 13 vídeos. 


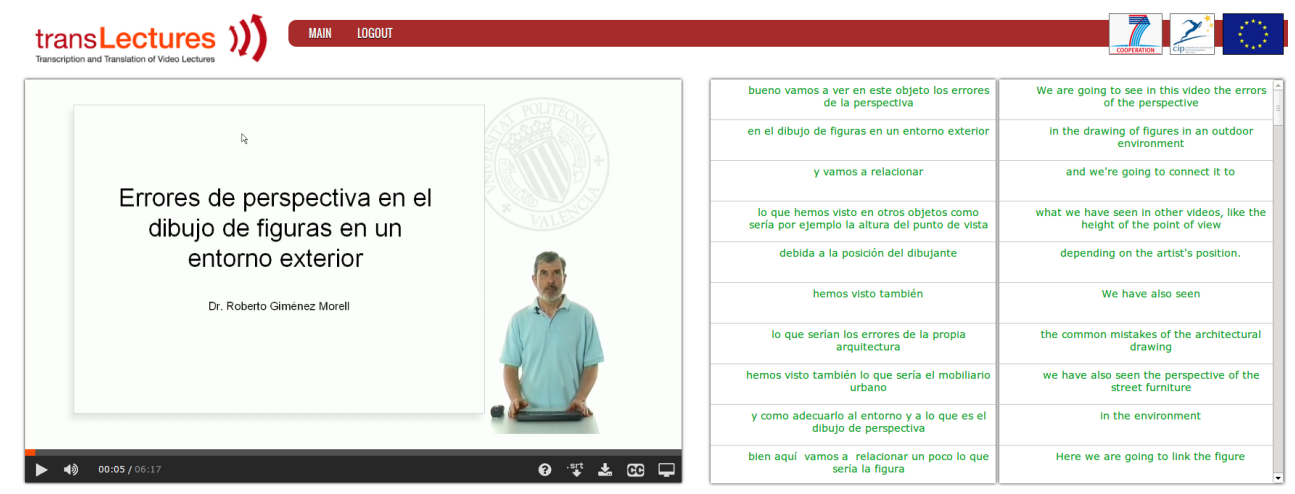

Fig. 2: Reproductor de vídeo transLectures empleado para las revisiones.

\section{Resultados}

Tras realizar un estudio comparativo entre la generación manual de subtítulos, y la revisión de subtítulos generados automaticamente, se ha confirmado una reducción significativa de dedicación temporal para las transcripciones en español, catalán e inglés, y en traducciones de español a inglés, en vídeos docentes poliMedia.

Así pues, obtenemos reducciones de dedicación temporal del $73 \%$ en la revisión de transcripciones en español, en comparación a realizarlas desde cero. Por otro lado, en el caso del inglés obtenemos reducciones del $38 \%$ de tiempo, y en el caso de la revisión de transcripciones de catalán la reducción temporal es del 44\%. Así pues, en cualquier caso la mejora obtenida por nuestros sistemas y presentada en este artículo es significativa y muy satisfactoria. Por otro lado, destacar que en el caso de las transcripciones en español el error de las mismas es del $12 \%$, por lo que pueden ser empleadas sin revisión si no se requiere de una transcripción perfecta.

Por otro lado, en el caso de la revisión de traducciones automáticas del español al inglés, la reducción temporal, respecto a hacerlo desde cero desde las transcripciones en español, es del $65 \%$. Esto reafirma la conveniencia de realizar la revisión de traducciones generadas automticamente para obtener traducciones perfectas con una dedicación temporal menor.

\section{Conclusiones}

En este artículo hemos presentado una evaluación de un sistema de subtitulación de vídeos docentes. En esta evaluación participaron profesores de la UPV en el marco del programa Docència en Xarxa 2013-2014. Este sistema de subtitulación ha sido integrado en poliMedia posibilitando la revisión de la transcripción o traducción de cualquier vídeo disponible. 
La subtitulación automatica de vídeos docentes en diferentes idiomas es una herramienta poderosa, que permite una revisión sencilla y rápida de los subtítulos, y que aporta un importante valor añadido a los mismos con un coste muy inferior a su generación completamente manual. De esta forma, este artículo ejemplifica el uso eficiente y seguro de las técnicas de reconocimiento de voz y traducción automáticas en entornos reales sobre grandes repositorios de vídeos institucionales, como es el caso de poliMedia en la UPV.

\section{Referencias bibliográficas}

Silvestre, Joan Albert y col. (2012). "transLectures". En: Proceedings of IberSPEECH 2012.

Turró, Carlos y col. (2009). "Polimedia: a system for successful video e-learning". En: Proc. of EUNIS.

Valor Miró, Juan Daniel y col. (2012). "Integrating a State-of-the-Art ASR System into the Opencast Matterhorn Platform". En: Advances in Speech and Language Technologies for Iberian Languages. Vol. 328. CCIS. Springer, págs. 237-246. ISBN: 978-3-642-35291-1.

Valor Miró, Juan Daniel y col. (2014a). "Evaluación del proceso de revisión de transcripciones automáticas para vídeos Polimedia". En: I Jornadas de Innovación Educativa y Docencia en Red (IN-RED 2014). Universitat Politècnica de València, págs. 272-278.

- (2014b). "Evaluating intelligent interfaces for post-editing automatic transcriptions of online video lectures". En: Open Learning: The Journal of Open, Distance and e-Learning 29.1, págs. 72-85. 